\title{
MCAs in Arabidopsis are Ca2+-permeable mechanosensitive channels innately sensitive to membrane tension
}

Kenjiro Yoshimura

Shibaura Institute of Technology https://orcid.org/0000-0003-3661-0890

Kazuko lida

Tokyo Gakugei University

Hidetoshi lida ( $\nabla$ iida@u-gakugei.ac.jp )

Tokyo Gakugei University https://orcid.org/0000-0002-3986-2615

\section{Article}

Keywords: Mechanical Stress, Artificial Liposome Membranes, Flurometry, Patch-clamp Technique

Posted Date: January 5th, 2021

DOI: https://doi.org/10.21203/rs.3.rs-126545/v1

License: (1) This work is licensed under a Creative Commons Attribution 4.0 International License.

Read Full License

Version of Record: A version of this preprint was published at Nature Communications on October 19th, 2021. See the published version at https://doi.org/10.1038/s41467-021-26363-z. 
MCAs in Arabidopsis are $\mathrm{Ca}^{2+}$-permeable mechanosensitive channels innately sensitive to membrane tension

Kenjiro Yoshimura ${ }^{1 *}$, Kazuko Iida ${ }^{2,3}$, and Hidetoshi Iida ${ }^{2 *}$

${ }^{1}$ Department of Machinery and Control Systems, College of Systems Engineering and Science, Shibaura Institute of Technology, Saitama 337-8570, Japan

${ }^{2}$ Department of Biology, Tokyo Gakugei University, 4-1-1 Nukuikita-machi, Koganei, Tokyo 184-8501, Japan

${ }^{3}$ Laboratory of Biomembrane, Tokyo Metropolitan Institute of Medical Science, 2-1-6 Kamikitazawa, Setagaya, Tokyo 156-8506, Japan

*Co-corresponding authors

E-mail: kenjiroy@shibaura-it.ac.jp; iida@u-gakugei.ac.jp 
Mechanosensitive (MS) ion channels respond to mechanical stress and convert it to electric and ionic signals that activate appropriate cellular mechanisms. Although the force-sensing mechanisms of MS channels remain obscure, the following have been proposed: activation by force from membrane lipids and activation by force delivered from associated proteins. Five MS channel families have been identified to date in plants, including the Arabidopsis thaliana Mid1-Complementing Activity (MCA) channel; however, their activation mechanisms have not yet been elucidated in detail. We herein demonstrated that the MCA2 channel is a $\mathrm{Ca}^{2+}$ permeable mechanosensitive channel that is directly activated by membrane tension. The N-terminal 173 residues of MCA1 and MCA2 were synthesized in vitro, purified, and reconstituted into artificial liposome membranes. $\mathrm{Ca}^{2+}$ fluorometry demonstrated that liposomes reconstituted with MCA1(1-173) or MCA2(1-173) mediated $\mathrm{Ca}^{2+}$ influx. The patch-clamp technique revealed that the application of pressure to the membrane reconstituted with MCA2(1-173) elicited channel currents. This channel was also activated by voltage. Blockers for mechanosensitive channels inhibited stretch, but not voltage, activation. Since MCA proteins are found exclusively in plants, these results suggest that MCA represents a plant-type MS channel that opens directly with membrane stretch.

\section{Introduction}

MS channels are integral membrane proteins that comprise a gated pore to mediate the flux of ions across membranes in response to extrinsic mechanical stress, such as touch, wind, gravity, osmotic pressure, and pathogen invasion, as well as intrinsic mechanical stress, including cell division and cellular growth ${ }^{1,2}$. Despite their 
importance in responses to mechanical stress, the precise mechanisms for the gating of the MS channel remain unclear. However, the following two general mechanisms have been proposed and widely accepted: the force-from-lipids mechanism ${ }^{3,4}$, in which MS channels act as direct mechanosensors of tension in the lipid bilayer; and the force-from filaments mechanism ${ }^{5,6}$, in which the extracellular matrix and/or cytoskeleton act as mediators of the force that pulls MS channels open.

Five families of MS channels have been reported to date in plants: Mid1Complementing Activity (MCA) proteins ${ }^{7,8}$, MscS-like (MSL) proteins ${ }^{9,10}$, Two Pore Potassium (TPK) channels ${ }^{11}$, Reduced hyperosmolality-induced $\left[\mathrm{Ca}^{2+}\right]_{\mathrm{i}}$ increase (OSCA) channels ${ }^{12}$ evolutionally related to DUF221 $1^{13,14}$, and Piezo channels ${ }^{15}$. MCA channels are unique in terms of structural and evolutional aspects. Arabidopsis MCA proteins have only a single transmembrane segment and assemble into a homotetramer to constitute a channel ${ }^{16-18}$, while the subunits of other plant MS channel families have multiple transmembrane segments and form multimers, including dimers (TPK), trimers (Piezo), pentamers (OSCA), and heptamers (MSL) ${ }^{19-21}$. Genes encoding MCA proteins are found exclusively in plants ${ }^{7,22}$, while those of other plant MS channel homologs are found in bacteria, protists, fungi (MSL), and animals (Piezo, OSCA, and TKP $)^{9,11,12,15,23,24}$. MCA1 and MCA2 are involved in the response to gravity in Arabidopsis hypocotyls ${ }^{25}$. Moreover, MCA1 is required for perceiving the hardness of soil and hypoosmotic stress ${ }^{7}$. The $\mathrm{N}$-terminal 173 residues, including the transmembrane segment and EF-hand-like motif, are sufficient for $\mathrm{Ca}^{2+}$ uptake activity when expressed in yeast cells ${ }^{17}$. Therefore, a $\mathrm{Ca}^{2+}$-permeable pore is likely be composed of the N-terminal 173 residues. 
The demonstration of direct activation by membrane tension requires the functional reconstitution of purified channels into an artificial lipid bilayer and activation by membrane stretch. Arabidopsis OSCA1.2 has been shown to be inherently mechanosensitive in this type of experiment ${ }^{26}$. MSL, Piezo, and TKP in plants have not been shown to be inherently mechanosensitive, although their counterparts in bacteria and animals are inherently mechanosensitive. The present study was performed to investigate whether MCA, which is unique to plants, has independently gained the capacity to be activated directly by membrane tension. To this end, we synthesized and MCA1 and MCA2 in vitro, reconstituted them into liposomes, and examined the $\mathrm{Ca}^{2+}$ permeability and electrophysiological properties. Here, we report a C-terminally truncated form of the MCA2 protein synthesized and purified in vitro acts as an inherent $\mathrm{Ca}^{2+}$-permeable mechanosensitive channel that directly senses membrane tension to open.

\section{Results}

\section{In vitro synthesis of MCA1 and MCA2 proteins}

MCA1 and MCA2 proteins were synthesized using a liposome-supplemented wheat germ cell-free translation system ${ }^{27}$. We initially attempted to synthesize and purify full-length MCA1 and MCA2 proteins fused C-terminally with a 6xHis tag, designated MCA-6H and MCA2-6H, respectively; however, since they underwent extensive aggregation, we were unable to solubilize them during subsequent purification steps. Therefore, we synthesized MCA1(1-173)-6H and MCA2(1-173)-6H (Fig. 1, line 1) and found that both proteins aggregated to a markedly lesser extent. Note that, to avoid lengthy names of the constructs, the abbreviation of $6 \mathrm{xHis}, 6 \mathrm{H}$, was not added to 
the constructs in the main text hereafter. The synthesized proteins were solubilized with 1\% $n$-dodecyl- $\beta$-D-maltopyranoside (DDM) (Fig. 1, line 2) and applied to a nickelnitrilotriacetic acid (Ni-NTA) column for one-step affinity purification. The eluate (Fig. 1, line 4) was then subjected to PD-10 desalting column chromatography to change the elution solution to a buffer suitable for the reconstitution of channels on liposomes. The purified proteins in the final preparation migrated in SDS-PAGE gels with a mobility corresponding to a molecular mass of approximately $19 \mathrm{kDa}$, consistent with the expected protein size (Fig. 1, lane 5). The final preparation had no visible contaminant protein, except for a small amount of a protein with a slightly slower mobility, which always appeared with the same abundance ratio to the 19-kDa protein during the purification steps, suggesting that the slower mobility protein is a consequence of its anomalous SDS-PAGE behavior often caused by altered detergent binding to membrane $\operatorname{proteins}^{28}$.

\section{C-terminally truncated MCA1 and MCA2 are permeable to $\mathrm{Ca}^{2+}$}

Previous studies indicated that both MCA1(1-173) and MCA2(1-173) are permeable to $\mathrm{Ca}^{2+}$ in yeast cells ${ }^{17}$. Therefore, we investigated whether the two proteins synthesized in vitro permeated $\mathrm{Ca}^{2+}$ when incorporated into liposomes (hereafter referred to as MCA1(1-173) and MCA2(1-173) liposomes). The control group included liposomes prepared with the cell-free protein synthesis reaction products from the empty vector (vector liposome) and liposomes incubated without reaction products (pure liposome). Liposomes were loaded with the fluorescent $\mathrm{Ca}^{2+}$ indicator, fluo4, and fluorescence was monitored when $\mathrm{CaCl}_{2}$ was added to the bath solution. 
The bath solution initially contained $1 \mathrm{mM}$ EGTA, which chelates $\mathrm{Ca}^{2+}$. When the $\mathrm{CaCl}_{2}$ concentration was increased to $0.5 \mathrm{mM}$, a small increase in fluorescence was detected in the suspension of MCA1(1-173) and MCA2(1-173) liposomes (Fig. 2a). An elevation to $1.5 \mathrm{mM} \mathrm{CaCl}_{2}$ resulted in a larger increase in fluorescence. A still further elevation to $2.5 \mathrm{mM}$ did not evoke a marked increase in fluorescence. In contrast, only a small and gradual increase in fluorescence was observed in the vector and pure liposomes. The significantly larger increases observed in fluorescence in MCA1(1-173) and MCA2(1-173) liposomes than in control liposomes indicated an elevation in $\mathrm{Ca}^{2+}$ concentrations by the influx of $\mathrm{Ca}^{2+}$ through MCA1(1-173) and MCA2(1-173). The largest increase between 0.5 and $1.5 \mathrm{mM}$ was consistent with the $1: 1$ stoichiometry of the binding of $\mathrm{Ca}^{2+}$ to EGTA.

The site-directed mutagenesis of Asp-21, which is present in the transmembrane segments of both MCA1(1-173) and MCA2(1-173), showed that the D21N mutation resulted in the loss of $\mathrm{Ca}^{2+}$ uptake when expressed in yeast cells ${ }^{17}$. To clarify whether this defect in vivo correlated with $\mathrm{Ca}^{2+}$ influx into liposomes, we generated MCA1(1173)D21N and MCA2(1-173)D21N by cell-free synthesis. When MCA1(1-173)D21N and MCA2(1-173)D21N were reconstituted into liposomes, the changes observed in fluorescence upon increases in the bath $\mathrm{Ca}^{2+}$ concentration almost coincided with those in the pure liposome (Fig. 2b). Based on the results that a $\mathrm{Ca}^{2+}$ concentration increase was observed in MCA1(1-173) and MCA2(1-173) liposomes, but not in control liposomes, and that the increase was not observed in MCA1(1-173)D21N and MCA2(1173)D21N liposomes, we suggest that both MCA1(1-173) and MCA2(1-173) have the ability to mediate $\mathrm{Ca}^{2+}$ influx. 


\section{C-terminally truncated MCA2 is an inherently mechanosensitive channel}

The channel activities of MCA2(1-173) were examined using a patch clamp technique. A large blister of a lipid bilayer was generated from the liposomes reconstituted with MCA2(1-173). When $+160 \mathrm{mV}$ was applied to the membrane excised from the blister, sparse square wave currents with a constant amplitude were detected (Fig. 3a). When the holding potential was raised to $+180 \mathrm{mV}$ or higher, we observed increases in the frequency of the occurrence of current as well as the unit amplitude. This current was not observed when liposomes were prepared from the reaction product from an empty vector. The amplitude of currents nearly doubled when two square wave currents appeared to overlap (Fig. 3d). Since current followed a two-state model of open and closed states, MCA2(1-173) should form an ion channel.

When the holding potential was changed between $-200 \mathrm{mV}$ and $+200 \mathrm{mV}$, channel openings were only observed at potentials larger than $+100 \mathrm{mV}$ and the open probability increased with voltage (Fig. 3b). Unit current increased almost linearly between $+140 \mathrm{mV}$ and $+200 \mathrm{mV}$ and the slope appeared to be shallower at lower potentials (Fig. 3c). Slope conductance changes with voltage have also been reported for other channels ${ }^{29}$.

The mechanosensitivity of MCA2(1-173) was examined by the application of negative pressure through the patch pipette. Channel activity was not detected in the absence of pressure at $+100 \mathrm{mV}$; however, when negative pressure was gradually increased, channel events were initiated (Fig. 3d). Channel opening appeared to occur more frequently at a high pressure. The resting level of activity was resumed when pressure was released. Pressure dependence was obtained by sectioning the current trace according to the pressure range with a bin of $10 \mathrm{mmHg}$. Open probability markedly 
increased at pressures between 30 and $70 \mathrm{mmHg}$ (Fig. 3e). Examinations at higher pressures were not possible because of membrane breakage.

These results indicated that the channel activity of MCA2(1-173) increased with voltage and membrane stretch. We then investigated whether voltage and stretch activation are separatable. We initially tested gadolinium, which affects mechanosensitive channel activation by changing the physical properties of the lipid bilayer $^{30}$. When gadolinium was present in the bath solution, pressure application did not markedly increase channel activity over that in its absence (Fig. 4a). Pressure dependence confirmed that gadolinium suppressed activation by pressure at concentrations of 10 and $100 \mu \mathrm{M}$ (Fig. 4b). In contrast, voltage activation was largely unaffected by gadolinium (Fig. 4c). We then examined the effects of the spider venom toxin, GsMT4, which affects the interaction between the lipid bilayer and mechanosensitive channels ${ }^{31}$. GsMT4 also affected stretch activation, but not voltage activation (Fig. 4d, e). Thus, stretch activation of MCA2(1-173) can be blocked separately from voltage activation and would require physical interaction with lipid bilayer.

MS channels that are activated by membrane tension can be activated by altering the intrinsic membrane curvature ${ }^{32,33}$. We applied lysophosphatidylcholine, an inverted cone-shaped lipid, to the patch membrane reconstituted with MCA2(1-173) and found that channel activity increased without the application of pressure (Fig. 4f).

\section{Discussion}

The results showing an increase in channel activity with pressure, inhibition by mechanosensitive channel blockers, and activation by changing the intrinsic membrane 
curvature support the idea that MCA2(1-173) is a MS channel that is directly activated by membrane tension without the help of accessory components, such as a cytoskeleton and an extracellular material. Direct activation by membrane tension has also been reported for other MS channels; however, MCA2(1-173), which constitutes a homotetramer as a subunit with a single transmembrane segment, represents the simplest form of MS channels. Since the transmembrane $\alpha$ helix of MCA2 is amphipathic, the hydrophilic side including Asp-21 may assemble to form a channel pore and the hydrophobic side interacts with membrane lipids ${ }^{17}$. The failure to detect the influx of $\mathrm{Ca}^{2+}$ in liposomes reconstituted with D21N MCA2(1-173) supports this view.

MCA2(1-173) was activated by a voltage of $120 \mathrm{mV}$ or higher, which we assume to correspond to a membrane potential of $-120 \mathrm{mV}$ or lower in vivo. This threshold is above the resting potential recorded in mesophyll cells $(-160$ to $-180 \mathrm{mV})$ and root cells (-180 mV) of Arabidopsis ${ }^{34,35}$. However, the spontaneous opening of MCA2(1$173)$ at the resting potential is unlikely to occur because MCA2(1-173) passes $\mathrm{Ca}^{2+}$, which activates various targets. We speculate that the threshold may be lower or voltage activation may be suppressed in full-length MCA2 or in vivo. Since pressure activated MCA2(1-173) at voltages at which voltage activation did not occur, it is possible that a natural key signal to open MCA2(1-173) is membrane tension.

The absence of channel currents at negative voltages in MCA2(1-173) indicates strong rectification in which current flows only in one direction. The mechanism for rectification has been studied extensively in inward rectifier potassium channels. The rectification in inward rectifiers is not a result from an inherent property of channel itself. Rather, rectification is due to channel blockade by cations, such as $\mathrm{Mg}^{2+}$ and polyamines ${ }^{36}$ ( $\mathrm{Lu}$ et al, 2004). $\mathrm{Mg}^{2+}$ in the patch clamp solution used in this study is 
possible to contribute to the rectification. The observation of rectification also indicates that MCA2(1-173) proteins were incorporated into the liposome lipid bilayer in a unidirectional orientation and not in a directionally fifty-fifty manner. The directional incorporation into liposomes is not surprising since membrane proteins including ion channels have been known to be incorporated in a uniform direction when the dehydration/rehydration method is adopted for proteoliposome preparations. For instance, a bacterial mechanosensitive channel, $\mathrm{MscS}$, is incorporated in a $100 \%$ rightside-out configuration ${ }^{37}$. Various ion channels such as TREK-1, KirBAC1.1, and TRPV1 show rectification when reconstituted into liposomes, indicating that these channels are incorporated into membrane in a unidirectional manner ${ }^{38-40}$.

It currently remains unclear why $\mathrm{Ca}^{2+}$ flowed into liposomes in fluorescence experiments in the absence of pressure and voltage. Since the majority of liposomes that form after 30 minutes of sonication have a diameter smaller than $100 \mathrm{~nm}^{41}$, the curvature of liposomes may be sufficient to activate MCA2(1-173) because changes in the membrane curvature induced by the application of lysophosphatidylcholine activated MCA2(1-173) (Fig. 4f).

In summary, we showed that artificially synthesized MCA2(1-173) proteins formed an MS channel in liposome membranes in vitro. Purified MCA1(1-173) proteins may exhibit similar activity because the two truncated proteins represented essentially the same activity in the in vitro assay system (Fig. 2) and in yeast cells ${ }^{17}$. This is the first study to demonstrate that plant-specific membrane proteins self-assemble to become an active ion channel. Consequently, the coiled-coil and PLAC8 motifs, which are absent from MCA1(1-173) and MCA2(1-173), do not appear to be necessary for the formation and activity of MS channels and, thus, may be involved in the cellular 
modulation of MCA channels because they are present in the cytoplasmic side ${ }^{18}$. Therefore, the present study will serve as a starting point for obtaining a deeper understanding of plant MS channels in terms of structural and regulatory viewpoints.

\section{Methods}

In vitro synthesis and purification of MCA proteins. MCA1, MCA2, and their derivatives were prepared as follows. We explained the procedure for the MCA2(1-173) protein as a representative for simplicity. MCA2(1-173) fused C-terminally with a 6xHis tag (-MGSHHHHHH), designated MCA2(1-173)-6H, was synthesized in vitro using the ProteoLiposome Expression Kit (CellFree Sciences Co., Ltd, No. CFS-TRIPLE, Ehime, Japan) according to the manufacturer's protocol. A DNA fragment encoding MCA2(1-173)-6H was inserted between the EcoRV and NotI restriction sites of the in vitro expression vector, pEU-E01-MCS (CellFree Sciences Co., Ltd.). A translation reaction was conducted at $15^{\circ} \mathrm{C}$ for $20 \mathrm{~h}$ in the presence of asolectin liposomes. The crude ProteoLiposome fraction containing synthesized MCA2(1-173)$6 \mathrm{H}$ proteins was isolated by centrifugation at $20,000 \mathrm{~g}$ at $4^{\circ} \mathrm{C}$ for $10 \mathrm{~min}$, and washed four times with PBS by centrifugation. The final precipitate of the PBS wash from a 1$\mathrm{ml}$ translation reaction mixture was suspended in $1.5 \mathrm{ml}$ of solubilization buffer $(50 \mathrm{mM}$ sodium phosphate buffer, $\mathrm{pH}$ 8.0, 0.3 $\mathrm{M} \mathrm{NaCl}, 1 \% \mathrm{DDM}$, and $50 \mathrm{mM}$ imidazole), and centrifuged as described above. A portion $(0.5 \mathrm{ml})$ of the supernatant (a total of $1.5 \mathrm{ml}$ ) was applied three times to a Ni-NTA-Spin Column (QIAGEN, No. 31014, Hilden, Germany), and the column was washed with $0.6 \mathrm{ml}$ of solubilization buffer four times. MCA2(1-173)-6H proteins were eluted from the column with $0.6 \mathrm{ml}$ of elution buffer (50 mM sodium phosphate buffer, $\mathrm{pH} 8.0,0.3 \mathrm{M} \mathrm{NaCl}, 1 \%$ DDM, $0.3 \mathrm{M}$ imidazole), 
and the buffer was changed to MOPS buffer (5 mM MOPS-KOH, $\mathrm{pH} 7.2,0.2 \mathrm{M} \mathrm{KCl}$, 1\% DDM) using a PD-10 column (GE Healthcare Japan, No. 17-0851-01, Tokyo, Japan). The amount and purity of proteins were assessed by SDS-PAGE followed by silver staining. Typically, 60 80 $\mu \mathrm{g}$ of the pure MCA2(1-173)-6H protein was obtained from a 1-ml translation reaction mixture.

The same procedure was applied to the purification of MCA1(1-173)-6H, MCA1(1-173)D21N-6H, and MCA2(1-173)D21N-6H.

\section{Measurement of $\mathrm{Ca}^{2+}$ influx into liposomes}

Synthesized MCA1 and MCA2 were reconstituted into liposomes as described previously $^{42,43}$. Twenty milligrams of L- $\alpha$-phosphatidylcholine (Sigma, P5638) was dissolved with $0.5 \mathrm{~mL}$ of chloroform and the film was dried for one hour onto a glass test tube with nitrogen gas. The lipid film was kept in a vacuum for more than one hour. One milliliter of dehydration/rehydration (D/R) buffer $(200 \mathrm{mM} \mathrm{KCl}, 5 \mathrm{mM}$ MOPS$\mathrm{KOH}, \mathrm{pH}$ 7.2) was added and the suspension was vortexed until no particles were visible. The suspension was clarified with a bath sonicator at $30^{\circ} \mathrm{C}$ for 10 minutes.

The channel preparation was mixed with $9.2 \mathrm{mg}$ lipid at a protein-lipid ratio of 1:1,000. The mixture was supplemented with EGTA-KOH, $\mathrm{pH} 7.4$ (1 mM final concentration), n-octyl- $\beta$-D-glucoside (1\%), and Fluo $4(0.03 \mu \mathrm{M})$. Approximately 400 mg of Bio-Beads SM-2 Adsorbents (BioRad, Hercules, CA, USA) prehydrated in wash buffer (D/R buffer supplemented with 1 mM EGTA-KOH, pH7.4) was added to the sample and the mixture was incubated for 3 hours. The liquid fraction was taken and incubated with fresh Bio-Beads overnight. The fluorescent dye that was not incorporated in liposomes was removed by exchanging the buffer with wash buffer 
using a desalting column (Zeba Spin Desalting Column, 7K MWCO, Thermo Scientific, Rockford, IL, USA).

The fluorescence of Fluo4 incorporated into liposomes was measured with a fluorescence spectrometer (PerkinElmer LS55, Waltham, MA, USA). Excitation and emission wavelengths were 495 and $527 \mathrm{~nm}$, respectively (slit width: $5 \mathrm{~nm}$ ). $\mathrm{Ca}^{2+}$ concentrations were increased by sequentially adding $\mathrm{CaCl}_{2}$ solution. Liposomes were lysed by adding $1 \%$ (final concentration) n-octyl- $\beta$-D-glucoside at the end of measurements to obtain a measure of the total amount of Fluo- 4 and normalize the measurements.

\section{Patch clamp experiments on liposomes}

A lipid suspension was prepared as described above. The channel preparation was mixed with $5 \mathrm{mg}$ lipid at a protein-lipid ratio of 1:1,000. n-Octyl- $\beta$-D-glucoside was added to yield a final concentration of $1 \%$. The sample was incubated at $4^{\circ} \mathrm{C}$ on a seesaw shaker for one hour. Approximately $400 \mathrm{mg}$ of Bio-Beads prehydrated in D/R buffer was added to the sample and incubated for 3 hours. The liquid fraction was taken and incubated with fresh Bio-Beads overnight. The liquid fraction was ultracentrifuged at $\sim 160,000 \mathrm{~g}$ for 60 minutes. The pellet was suspended in $50 \mu \mathrm{L}$ dehydration buffer consisting of $10 \mathrm{mM}$ MOPS-KOH, pH 7.2, and 5\% ethylene glycol. The suspension was spotted on an 8-well slide and dehydrated under a vacuum at $4^{\circ} \mathrm{C}$ overnight. Between 10 and $20 \mu \mathrm{l}$ of $\mathrm{D} / \mathrm{R}$ buffer was placed onto the spots and kept in a moist chamber for more than one hour. The rehydrated preparation was placed in patch clamp buffer $(200 \mathrm{mM}$ $\mathrm{KCl}, 90 \mathrm{mM} \mathrm{MgCl} 2,10 \mathrm{mM} \mathrm{CaCl}_{2}, 5 \mathrm{mM}$ Hepes, $\mathrm{pH}$ 6.0) and allowed to form blisters. 
The blister membrane was caught onto a glass pipette and excised from the blister. The pipette contained the same buffer as the bath solution. The holding potential was controlled and the current was amplified with a patch clamp amplifier (Axopatch 200B, Axon Instruments, Union City, CA, USA). Current recordings were filtered at $2 \mathrm{kHz}$ and digitized at $5 \mathrm{kHz}$ using a Digidata 1322A interface with pCLAMP 9 software (Axon Instruments). Negative pressure was applied to the patch membrane through the pipette using a High-Speed Pressure Clamp-1 apparatus (HSPC-1; ALA Scientific Instruments, Westbury, NY, USA).

\section{Statistical analysis}

Two-sided $t$-test was applied where statistical analysis was performed. Equal variance was not assumed.

\section{Data availability}

The data that support all experimental findings of this study are available within the paper.

\section{References}

1. Peyronnet, R., Tran, D., Girault, T. \& Frachisse, J.-M. Mechanosensitive channels: feeling tension in a world under pressure. Front. Plant Sci. 5:558. doi: 10.3389/fpls.2014.00558 (2014).

2. Basu, D. \& Haswell, E. S. Plant mechanosensitive ion channels: an ocean of possibility. Curr. Opin. Plant Biol. 40, 43-48 (2017)

3. Martinac, B., Adler, J., \& Kung, C. Mechanosensitive ion channels of E. coli activated by amphipaths Nature 348, 261-263 (1990).

4. Teng, J., Loukin, S., Aniskin, A. \& Kung, C. The force-from-lipid (FFL) principle of mechanosensitivity, at large and in elements. Pflugers Arch. 467, 27-37 (2015). 
5. Chalfie, M. Neurosensory mechanotransduction. Nat. Rev. Mol. Cell Biol. 10, 44-52 (2009).

6. Katta, S., Krieg, M., \& Goodman, M. B. Feeling force: physical and physiological principles enabling sensory mechanotransduction. Annu. Rev. Cell Dev. Biol. 31, 347-371 (2015).

7. Nakagawa, Y., Katagiri, T., Shinozaki, K., Qi, Z., Tatsumi, H., Furuichi, T., Kishigami, A., Sokabe, M., Kojima, I., Sato, S., Kato, T., Tabarta, S., Iida, K., Terashima, A., Nakano, M., Ikeda, M., Yamanaka, T. \& Iida, H. (2007) Arabidopsis plasma membrane protein crucial for $\mathrm{Ca}^{2+}$ influx and touch sensing in roots. Proc. Natl. Acad. Sci. U S A 104, 3639-3644 (2007).

8. Furuichi, T., Iida, H., Sokabe, M., \& Tatsumi, H. Expression of Arabidopsis MCA1 enhanced mechanosensitive channel activity in the Xenopus laevis oocyte plasma membrane. Plant Signal. Behav. 7, 1022-1026 (2012).

9. Haswell, E. S., Peyronnet, R., Barbier-Brygoo, H., Meyerowitz, E. M. \& Frachisse, J.-M. Two MscS homologs provide mechanosensitive channel activities in the Arabidopsis root. Curr. Biol. 18, 730-734 (2008).

10. Maksaev, G. \& Haswell, E. S. MscS-Like10 is a stretch-activated ion channel from Arabidopsis thaliana with a preference for anions. Proc. Natl. Acad. Sci. USA 109, 19015-19020 (2012).

11. Maathuis, F. J. M. Vacuolar two-pore $\mathrm{K}^{+}$channels act as vacuolar osmosensors. New Phytol. 191, 84-91 (2011).

12. Yuan, F., Yang, H., Xue, Y., Kong, D., Ye, R., Li, C., Zhang, J., Theprungsirikul, L., Shrift, T., Krichilsky, B., Johnson, D. M., Swift, G. B., He, Y., Siedow, J. N. \& Pei, Z. M. OSCA1 mediates osmotic-stress-evoked $\mathrm{Ca}^{2+}$ increases vital for osmosensing in Arabidopsis. Nature 514, 367-371 (2014).

13. Hou, C., Tian, W., Kleist, T., He, K., Garcia, V., Bai, F., Hao, Y., Luan, S., \& Li, L. DUF221 proteins are a family of osmosensitive calcium-permeable cation channels conserved across eukaryotes. Cell Res. 24, 632-635 (2014).

14. Medrano-Soto, A., Moreno-Hagelsieb, G., McLaughlin, D., Ye, Z. S., Hendargo, K. J. \& Saier, M. H., Jr. Bioinformatic characterization of the anoctamin superfamily of $\mathrm{Ca}^{2+}$-activated ion channels and lipid scramblases. PLoS ONE 13(3): e0192851. doi.org/10.1371/journal.pone.0192851 (2018).

15. Mousavi, S. A. R., Dubin, A. D., Zeng, W.-Z., Coombs, A. M., Do, K., Ghadiri, D. A., Ge, C., Zhao, Y. \& Patapoutian, A. PIEZO ion channel is required for root mechanotransduction in Arabidopsis thaliana. bioRxiv. doi.org/10.1101/2020.08.27.270355 (2020). 
16. Kamano, S., Kume, S., Iida, K., Lei, K.-J., Nakano, M. \& Iida, H. Transmembrane topologies of $\mathrm{Ca}^{2+}$-permeable mechanosensitive channels MCA1 and MCA2 in Arabidopsis thaliana. J. Biol. Chem. 290, 30901-30909 (2015)

17. Nakano, M., Iida, K., Nyunoya, H. \& Iida, H. Determination of structural regions important for $\mathrm{Ca}^{2+}$ uptake activity in Arabidopsis MCA1 and MCA2 expressed in yeast. Plant Cell Physiol. 52, 1915-1930 (2011).

18. Shigematsu, H., Iida, K., Nakano, M., Chaudhuri, P., Iida, \& Nagayama, K. Structural characterization of the mechanosensitive channel candidate MCA2 from Arabidopsis thaliana. PLoS ONE 9(1): e87724. (2014). Doi:10.1371/journal.pone.0087724.

19. Wilson, M. E., Malsaev, G. \& Haswell, E. S. Msc-S-like mechanosensitive channels in plants and microbes. Biochemistry 52, 5708-5722 (2013)

20. Maity, K., Heumann, J. M., McGrath, A. P., Kopcho, N. J., Hsu, P.-K., Lee, C.-W., Mapes, J. H., Garza, D., Krishnan, S., Morgan, G. P., Hendargo, K. J., Klose, T., Rees, S. D., Medrano-Soto, A., Saier, M. H., Jr., Piñeros, M., Komives, E. A., Schroeder, J. I., Chang, G. \& Stowell M. H. B. Cryo-EM structure of OSCA1.2 from Oryza sativa elucidates the mechanical basis of potential membrane hyperosmolality gating. Proc. Natl. Acad. Sci. USA 116, 14309-14318 (2019)

21. Saotome, Kei., Murthy, S. E., Kefauver, J. M., Whitman, T., Patapoutian, A. \& Ward, A. B. Structure of the mechanically activated ion channel Piezo1. Nature 554, 481-486 (2018).

22. Kurusu, T., Kuchitsu, K., Nakano, M., Nakayama, Y. \& Iida, H. Plant mechanosensing and $\mathrm{Ca}^{2+}$ transport. Trends Plant Sci. 18, 227-233 (2013).

23. Nakayama, Y., Fujiu, K., Sokabe, M. \& Yoshimura, K. Molecular and electrophysiological characterization of a mechanosensitive channel expressed in the chloroplasts of Chlamydomonas. Proc. Natl. Acad. Sci. U.S.A. 104, 5883-5888 (2007).

24. Nakayama, Y., Yoshimura, K. \& Iida, H. Organellar mechanosensitive channels in fission yeast regulate the hypo-osmotic shock response. Nat Commun. 3: 1020 (2012).

25. Hattori, T., Otomi, Y., Nakajima, Y., Soga, K., Wakabayashi, K., Iida, H. \& Hoson, T. MCA1 and MCA2 are involved in the response to hypergravity in Arabidopsis hypocotyls. Plants (Basel) 9: 590. doi: 10.3390/plants9050590. (2020).

26. Murthy, S. E., Dubin, A. E., Whitwam, T., Jojoa-Cruz, S., Cahalan, S. M., Mousavi, S. A. R. , Ward, A. B. \& Patapoutian, A. OSCA/TMEM63 are an evolutionarily conserved family of mechanically activated ion channels. Elife 7:e41844. doi: 10.7554/eLife.41844. (2018). 
27. Nozawa, A., Ogasawara, T., Matsunaga, S., Iwasaki, T., Sawasaki, T. \& Endo, Y. Production and partial purification of membrane proteins using a liposome-supplemented wheat cell-free translation system. BMC Biotechnol. 11:35. doi: 10.1186/1472-6750-1135 (2011).

28. Rath, A., Glibowicka, M., Nadeau, V. G., Chen, G. \& Deber, C. M. Detergent binding explains anomalous SDS-PAGE migration of membrane proteins. Proc. Natl. Acad. Sci. USA 106, 1760-1765 (2009).

29. Mak, D. O., McBride, S., Raghuram, V., Yue, Y., Joseph, S. K. \& Foskett, J. K. Single-channel properties in endoplasmic reticulum membrane of recombinant type 3 inositol trisphosphate receptor. J. Gen. Physiol. 115, 241-256 (2000).

30. Ermakov, Y. A., Averbakh, A. Z., Yusipovich, A. I. \& Sukharev, S. Dipole potentials indicate restructuring of the membrane interface induced by gadolinium and beryllium ions. Biophys. J. 80, 1851-1862 (2001).

31. Suchyna, T. M. , Tape, S. E., Koeppe 2nd, R. E., Andersen, O. S., Sachs, F. \& Gottlieb, P. A. Bilayer-dependent inhibition of mechanosensitive channels by neuroactive peptide enantiomers. Nature 430, 235-240 (2004).

32. Martinac, B., Adler, J. \& Kung, C. Mechanosensitive ion channels of E. coli activated by amphipaths. Nature 348, 261-263 (1990).

33. Perozo, E., Cortes, D. M., Sompornpisut, P., Kloda, A. \& Martinac, B. Open channel structure of MscL and the gating mechanism of mechanosensitive channels. Nature 418, 942-948 (2002).

34. Shabala, S. N. \& Lew, R. R. Turgor regulation in osmotically stressed Arabidopsis epidermal root cells. Direct support for the role of inorganic ion uptake as revealed by concurrent flux and cell turgor measurements. Plant Physiol. 129, 290-299. (2002).

35. Reyer, A., Häßler, M., Scherzer, S., Huang, S., Pedersen, J. T., Al-Rascheid, K. A. S., Bamberg, E., Palmgren, M., Dreyer, I., Nagel, G., Hedrich, R. \& Becker, D.

36. Lu, Z. Mechanism of rectification in inward-rectifier $\mathrm{K}^{+}$channels. Annu. Rev. Physiol. 66, 103-129 (2004).

37. Nomura, T., Cox, C. D., Bavi, N., Sokabe, M. \& Boris Martinac, B. Unidirectional incorporation of a bacterial mechanosensitive channel into liposomal membranes. FASEB J. 29, 4334-4345 (2015).

38. Cheng, W. W., Enkvetchakul, D., \& Nichols, C. G. KirBac1.1: it's an inward rectifying potassium channel. J. Gen. Physiol. 133, 295-305 (2009).

39. Berrier, C., Pozza, A., de Lacroix de Lavalette, A., Chardonnet, S., Mesneau, A., Jaxel, C., le Maire, M., \& Ghazi, A. The purified mechanosensitive channel TREK-1 is directly sensitive to membrane tension. J. Biol. Chem. 288, 27307-27314 (2013). 
40. Cao, E., Cordero-Morales, J. F., Liu, B., Qin, F., \& Julius, D. TRPV1 channels are intrinsically heat sensitive and negatively regulated by phosphoinositide lipids. Neuron 77, 667-679 (2013).

41. Channelrhodopsin-mediated optogenetics highlights a central role of depolarizationdependent plant proton pumps. Proc. Natl. Acad. Sci. USA 117, 20920-20925 (2020).

42. Woodbury, D. J., Richardson, E. S., Grigg, A. W., Welling, R. D., \& Knudson, B. H. Reducing liposome size with ultrasound: bimodal size distributions. J. Liposome Res. 16, 57-80 (2006).

43. Blount, P., Sukharev, S., Moe, P., Martinac, B. \& Kung, C. Mechanosensitive channels of bacteria. Methods Enzymol. 294:458-482 (1999).

44. Yoshimura, K., Usukura, J. \& Sokabe, M. Gating-associated conformational changes in the mechanosensitive channel MscL. Proc. Natl. Acad. Sci. USA 105, 4033-4038 (2008).

\section{Acknowledgements}

This work was supported by a Grant-in-Aid for Scientific Research on Innovative Areas (25120708 to H.I.) from the Ministry of Education, Culture, Sports, Science and Technology, a Grant-in-Aid for Scientific Research (C) (17K07370 to K.Y.) from the Japan Society for Promotion of Science, and AMED/PRIME (JP18gm5810013 to K.Y.) from the Japan Agency for Medical Research and Development. We thank Ms. Aki Nakamura and Dr. Megumi Yoshida for technical assistance.

\section{Author Contributions}

H.I. conceived the research. K.Y., K.I. and H.I. designed the experiments. K.I. performed the experiments of protein synthesis and purification. K.Y. performed the experiments of fluorescence measurements and patch-clamp analyses. K.Y., K.I. and H.I. analyzed the data and wrote the manuscript. All authors read and approved the final manuscript.

\section{Competing interests}


The authors declare no competing interests.

\section{Figure legends}

Fig. 1 | Purification of MCA2(1-173)-6H. Protein samples were subjected to SDS-PAGE followed by silver staining. Lane 1, A crude ProteoLiposome fraction. Lane 2, 1\% DDMsolubilized sample. Lane 3, A flow-through fraction from a Ni-NTA column. Lane 4, An eluate from the same column eluted with $300 \mathrm{mM}$ imidazole. Lane 5, An eluate from a PD-10 desalting column eluted with MOPS buffer described in the Methods section. One-4000th of each sample was loaded onto the respective lanes.

\section{Fig. $2 \mid \mathrm{Ca}^{2+}$ permeation through MCA1 and MCA2 reconstituted into liposomes}

containing fluo4. $\mathrm{CaCl}_{2}$ was sequentially added to the bath solution containing $1 \mathrm{mM}$ EGTA. A detergent was added at the end of the experiment and fluorescence at this point was used to normalize fluorescence. (a) Fluorescence from liposomes reconstituted with MCA1 or MCA2 and control group liposomes. (b) Fluorescence from liposomes reconstituted with D21N MCA1(1-173) or D21N MCA2 (1-173). The mean and standard deviation of three measurements. In (a), $p$-values of $t$-test between the data from MCA1 or MCA2 and those from the liposome, a negative control, are indicated at $2.5 \mathrm{mM} \mathrm{CaCl}_{2}$.

\section{Fig. 3 | Channel activities of MCA2(1-173)-6H reconstituted in the lipid bilayer examined} by the patch clamp technique. (a) Channel current at a holding potential of 140 to $200 \mathrm{mV}$. (b) Changes in the open probability with the holding potential. (c) Changes in unit current with the holding potential. (d) Channel current (top trace) when a negative pressure (lower trace) was applied. The holding potential was $100 \mathrm{mV}$. (e) Changes in the open probability with the 
application of negative pressure. Data collected at 140 and $160 \mathrm{mV}$ were combined. In b, c, and e, the mean and standard deviation are shown in red. Small circles indicate individual data. Data from $17(\mathrm{~b}, \mathrm{c})$ and $6(\mathrm{e})$ patches.

Fig. 4 | Effects of mechanosensitive channel blockers on channel activities of MCA2(1173)-6H. (a) Negative pressure was applied before (left) and after (right) the application of 30 $\mu \mathrm{M} \mathrm{GdCl}_{3}$. Current (top trace) and pressure (lower trace) are shown. The holding potential was $140 \mathrm{mV}$. (b, c) Changes in the open probability with pressure (b) and voltage (c) when 0 (black), 10 (blue), or 100 (red) $\mu \mathrm{M} \mathrm{GdCl}_{3}$ was applied. (d, e) Changes in the open probability with pressure (d) and voltage (e) when 0 (black) or 5 (red) $\mu \mathrm{M}$ GsMT4 was applied. (f) Current traces before (left) and after (right) the application of lysophosphatidylcholine. The membrane was broken at the end of recording. The holding potential was $140 \mathrm{mV}$. In b, c, $\mathrm{d}$, and e, the mean and standard deviation are shown in red. Small circles indicate individual data. Data from 5 (b), 3 (c), 4 (d), and 3 (e) patches. In (b) and (d), $p$-values of $t$-test between the data obtained in the presence of blockers and those obtained in the absence are indicated. 


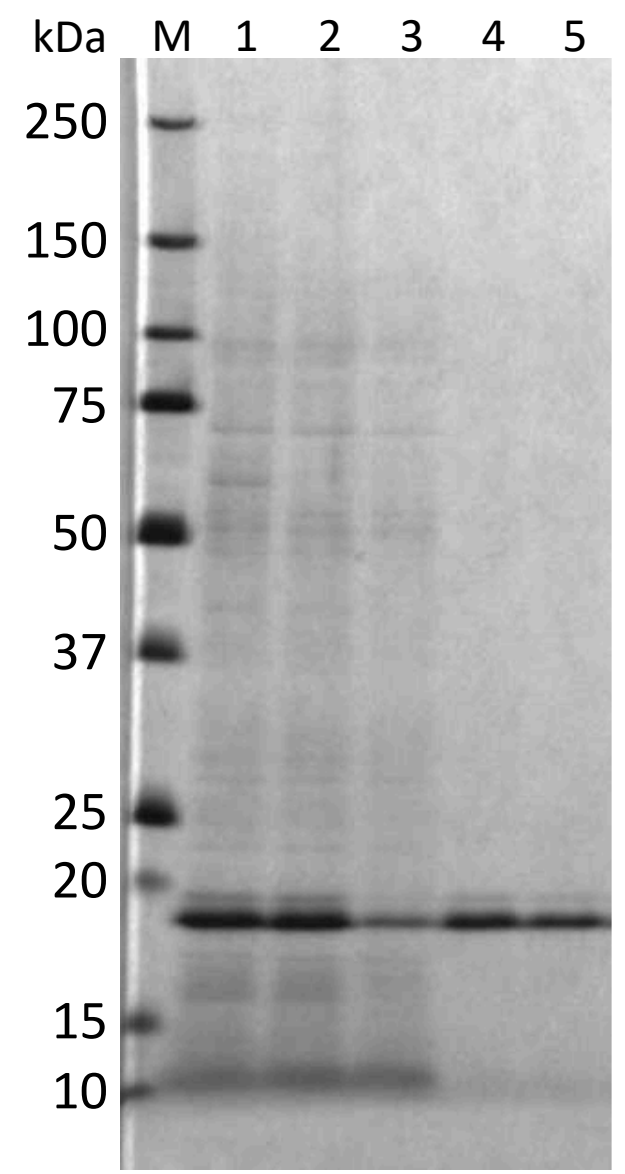

Fig. 1 | Purification of MCA2(1-173)-6H. Protein samples were subjected to SDS-PAGE followed by silver staining. Lane 1, A crude ProteoLiposome fraction. Lane 2, 1\% DDM-solubilized sample. Lane 3, A flow-through fraction from a Ni-NTA column. Lane 4, An eluate from the same column eluted with $300 \mathrm{mM}$ imidazole. Lane 5, An eluate from a PD-10 desalting column eluted with MOPS buffer described in the Methods section. One-4000th of each sample was loaded onto the respective lanes. 
a

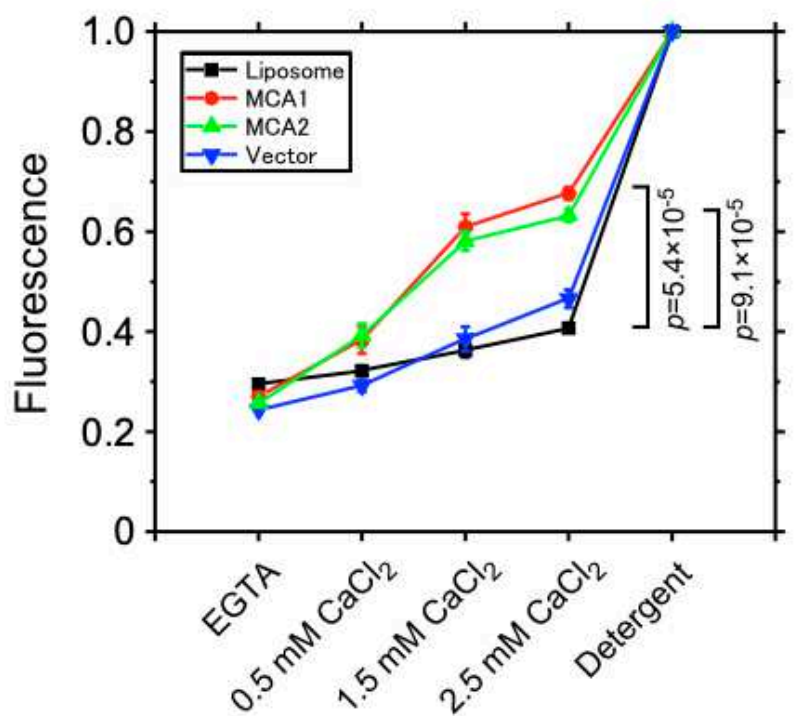

b

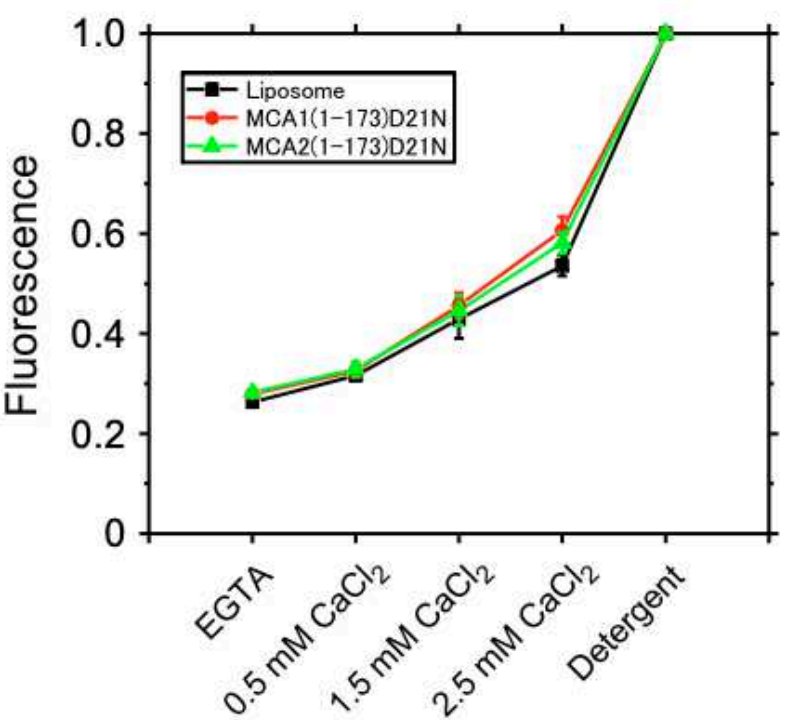

Fig. 2 | $\mathrm{Ca}^{2+}$ permeation through MCA1 and MCA2 reconstituted into liposomes containing fluo4. $\mathrm{CaCl}_{2}$ was sequentially added to the bath solution containing 1 mM EGTA. A detergent was added at the end of the experiment and fluorescence at this point was used to normalize fluorescence. (a) Fluorescence from liposomes reconstituted with MCA1 or MCA2 and control group liposomes. (b) Fluorescence from liposomes reconstituted with D21N MCA1(1-173) or D21N MCA2 (1-173). The mean and standard deviation of three measurements. In (a), $p$-values of $t$-test between the data from MCA1 or MCA2 and those from the liposome, a negative control, are indicated at $2.5 \mathrm{mM} \mathrm{CaCl}_{2}$. 
a

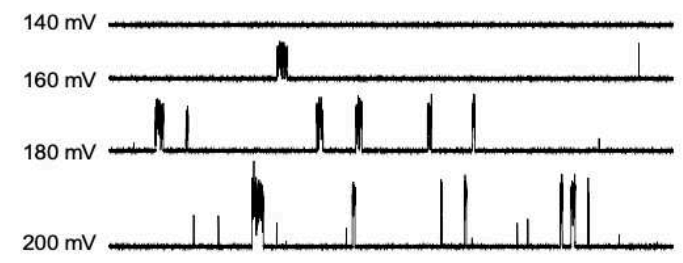

b

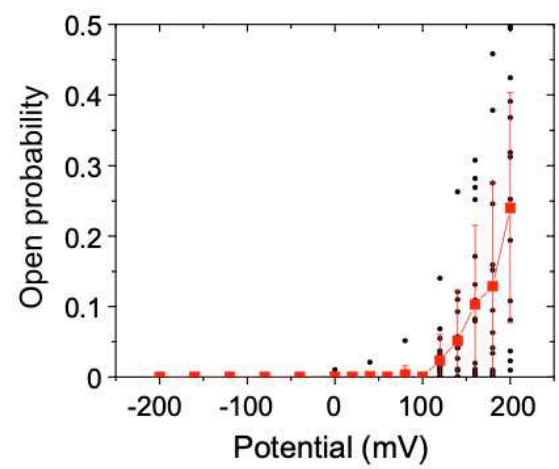

d
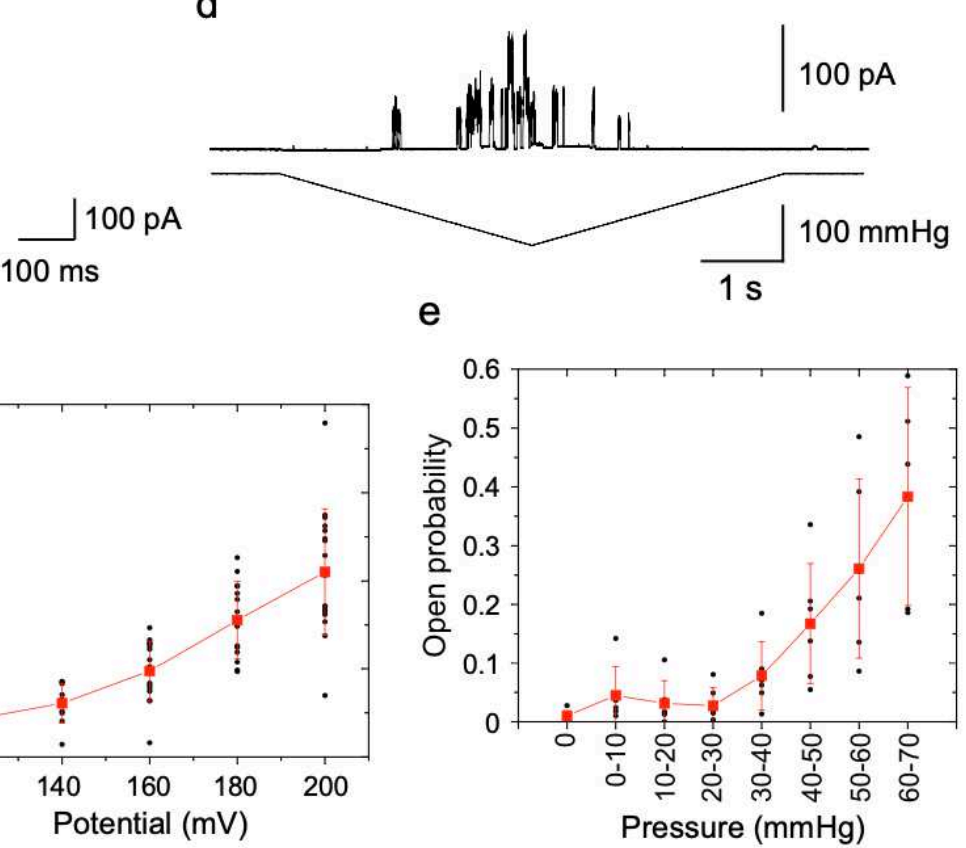

Fig. 3 | Channel activities of MCA2(1-173)-6H reconstituted in the lipid bilayer examined by the patch clamp technique. (a) Channel current at a holding potential of 140 to $200 \mathrm{mV}$. (b) Changes in the open probability with the holding potential. (c) Changes in unit current with the holding potential. (d) Channel current (top trace) when a negative pressure (lower trace) was applied. The holding potential was $100 \mathrm{mV}$. (e) Changes in the open probability with the application of negative pressure. Data collected at 140 and $160 \mathrm{mV}$ were combined. In b, c, and e, the mean and standard deviation are shown in red. Small circles indicate individual data. Data from 17 (b, c) and 6 (e) patches. 
a

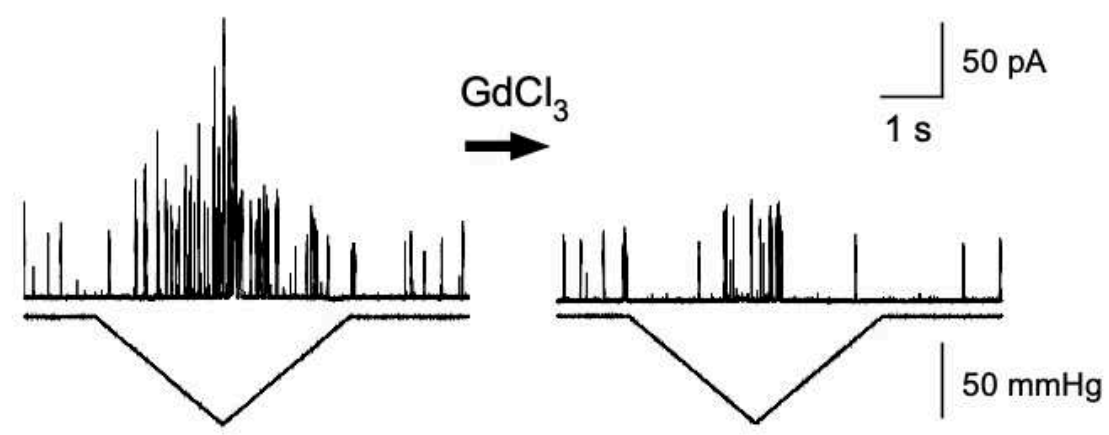

b

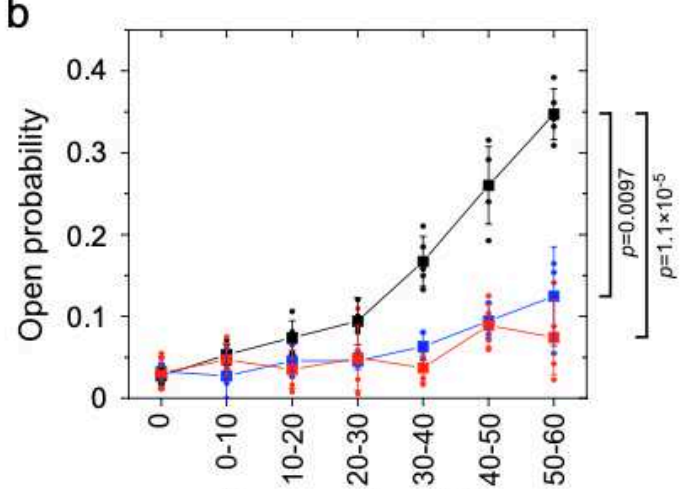

C

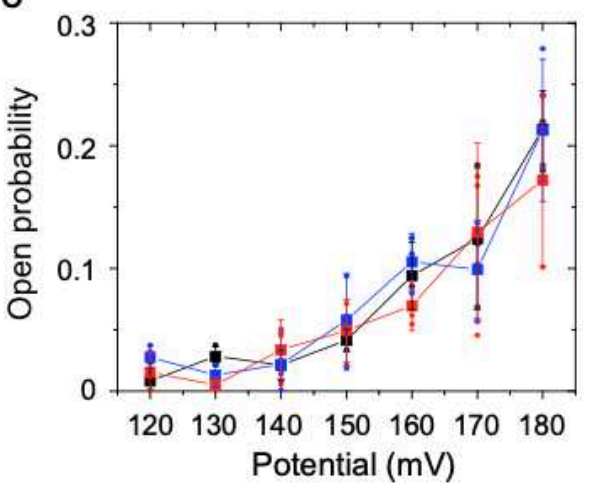

d

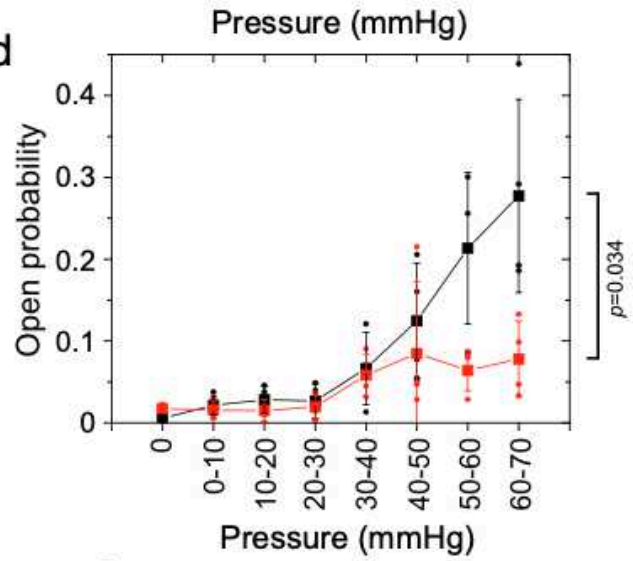

e

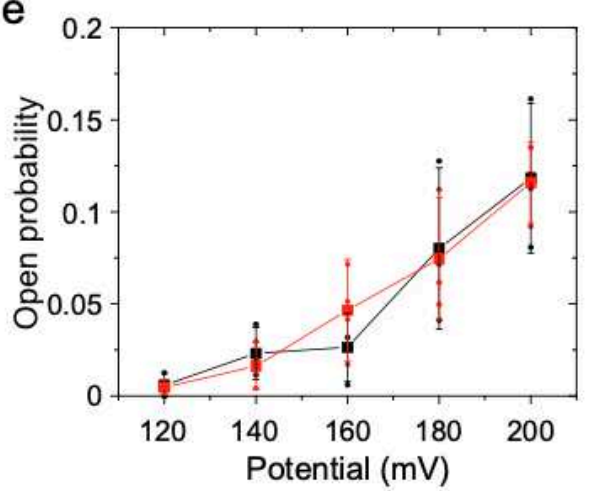

f

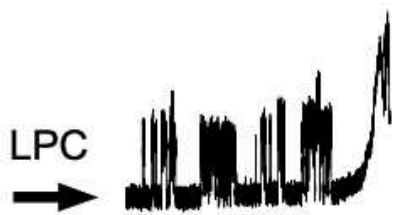

$\prod_{1 \mathrm{~s}} 50 \mathrm{pA}$

Fig. 4 | Effects of mechanosensitive channel blockers on channel activities of MCA2(1-173)-6H. (a) Negative pressure was applied before (left) and after (right) the application of $30 \mu \mathrm{M} \mathrm{GdCl}$. Current (top trace) and pressure (lower trace) are shown. The holding potential was $140 \mathrm{mV}$. (b, c) Changes in the open probability with pressure (b) and voltage (c) when 0 (black), 10 (blue), or 100 (red) $\mu \mathrm{M} \mathrm{GdCl}_{3}$ was applied. (d, e) Changes in the open probability with pressure (d) and voltage (e) when 0 (black) or 5 (red) $\mu \mathrm{M}$ GsMT4 was applied. (f) Current traces before (left) and after (right) the application of lysophosphatidylcholine. The membrane was broken at the end of recording. The holding potential was $140 \mathrm{mV}$. In b, c, d, and e, the mean and standard deviation are shown in red. Small circles indicate individual data. Data from 5 (b), 3 (c), 4 (d), and 3 (e) patches. In (b) and (d), $p$-values of $t$-test between the data obtained in the presence of blockers and those obtained in the absence are indicated. 


\section{Figures}

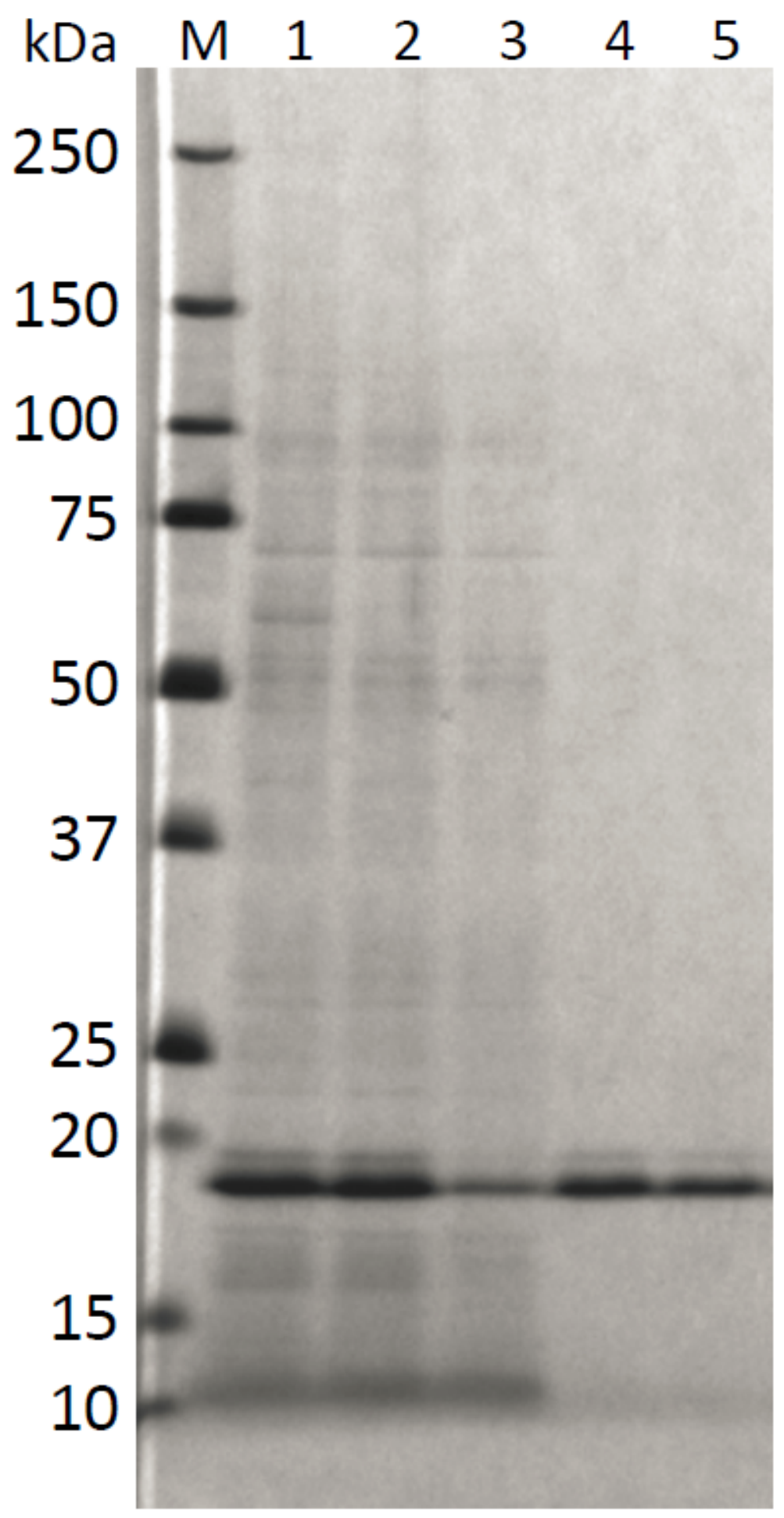

\section{Figure 1}

Purification of MCA2(1-173)-6H. Protein samples were subjected to SDS-PAGE followed by silver staining. Lane 1, A crude ProteoLiposome fraction. Lane 2, 1\% DDMsolubilized sample. Lane 3, A flow-through fraction from a Ni-NTA column. Lane 4, An eluate from the same column eluted with $300 \mathrm{mM}$ imidazole. 

section. One-4000th of each sample was loaded onto the respective lanes.

a

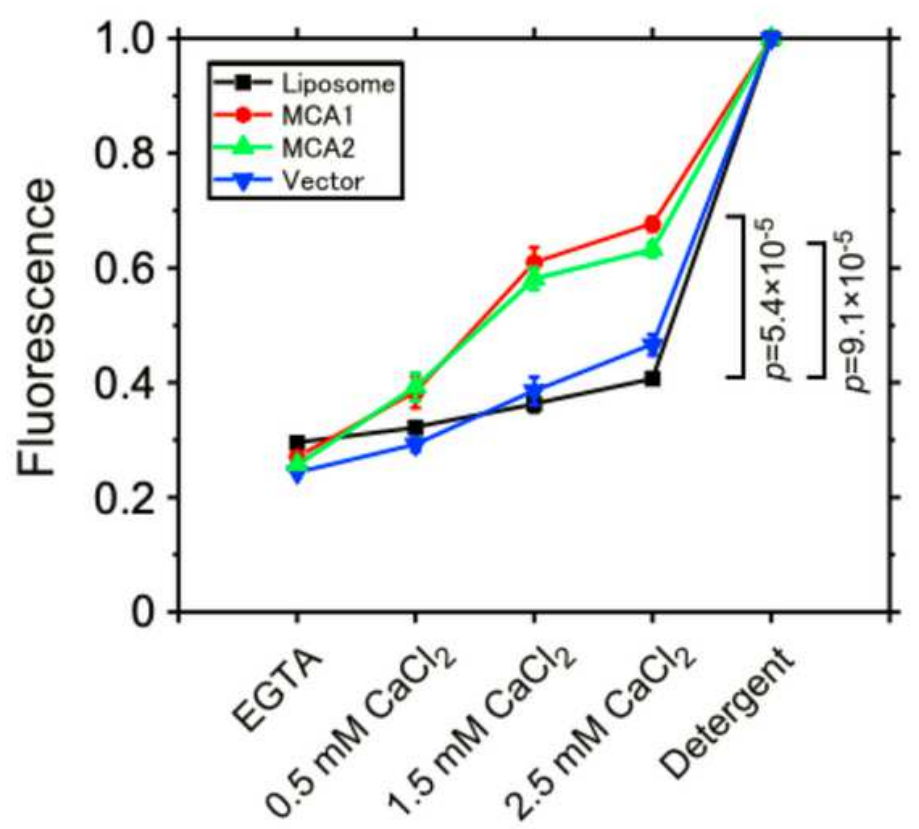

b

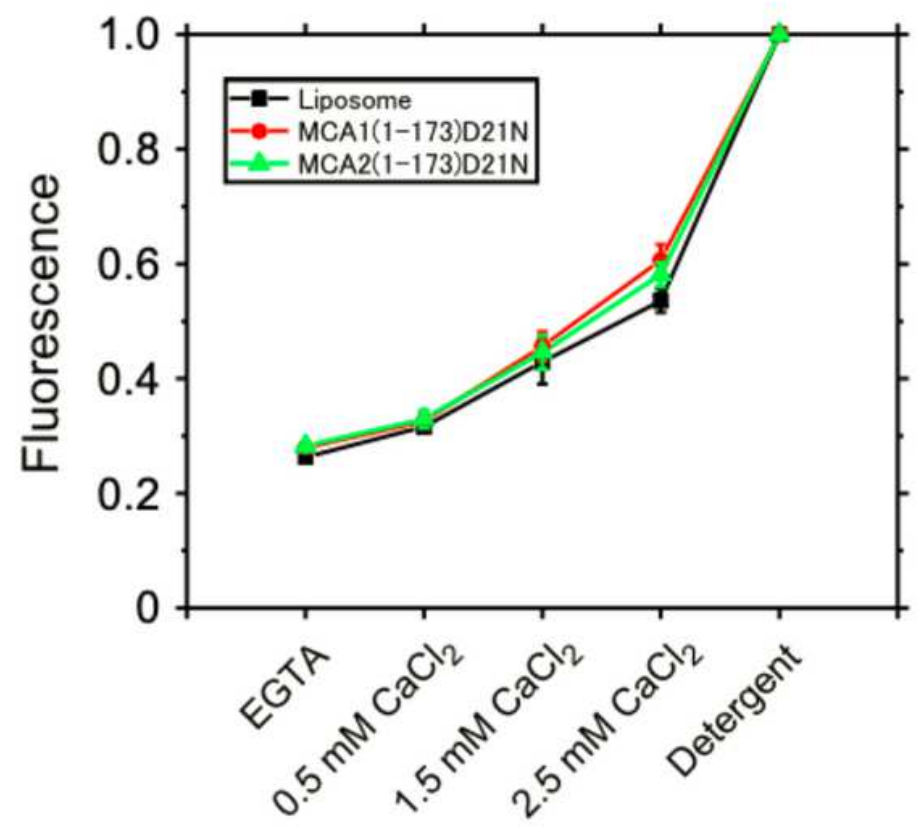

Figure 2

$\mathrm{Ca} 2+$ permeation through MCA1 and MCA2 reconstituted into liposomes containing fluo4. $\mathrm{CaCl} 2$ was sequentially added to the bath solution containing $1 \mathrm{mM} \mathrm{EGTA}$. A detergent was added at the end of the experiment and fluorescence at this point was used to normalize fluorescence. (a) Fluorescence from liposomes reconstituted with MCA1 or MCA2 and control group liposomes. (b) Fluorescence from liposomes reconstituted with D21N MCA1(1-173) or D21N MCA2 (1-173). The mean and standard deviation of three measurements. In (a), p-values of t-test between the data from MCA1 or MCA2 and those from the liposome, a negative control, are indicated at $2.5 \mathrm{mM} \mathrm{CaCl} 2$. 
a

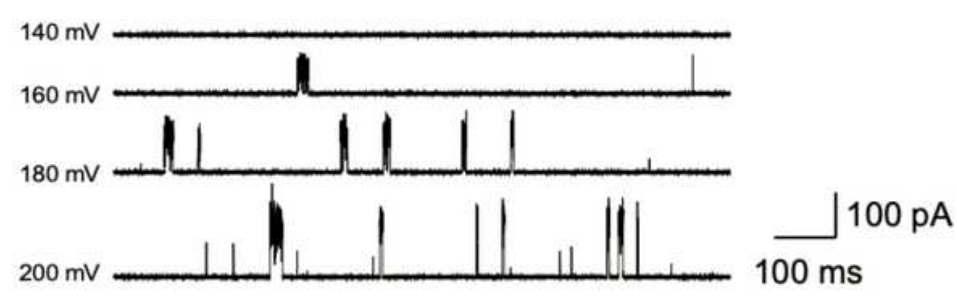

b

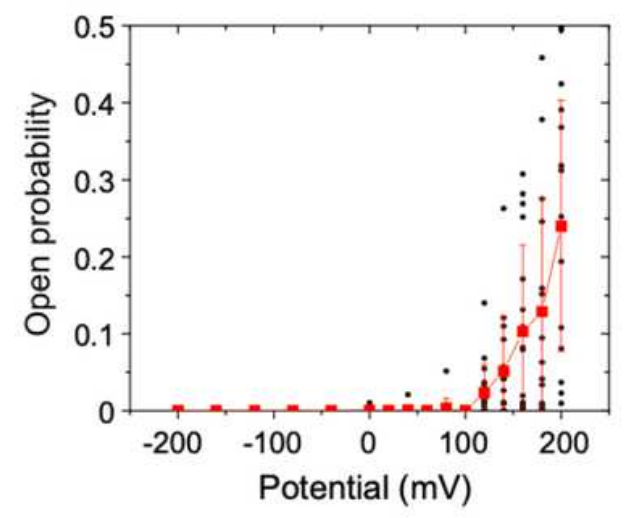

C

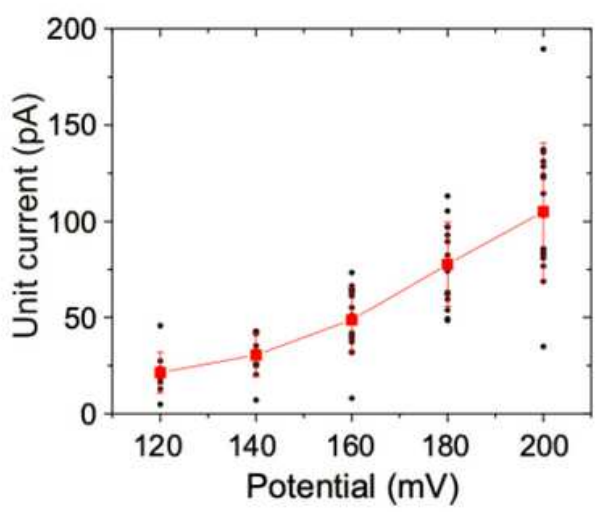

d

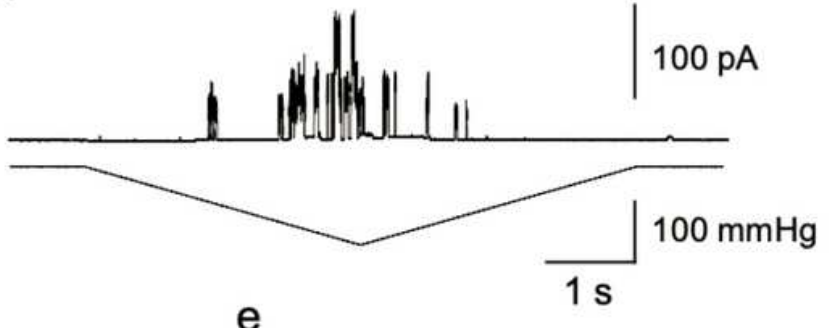

e

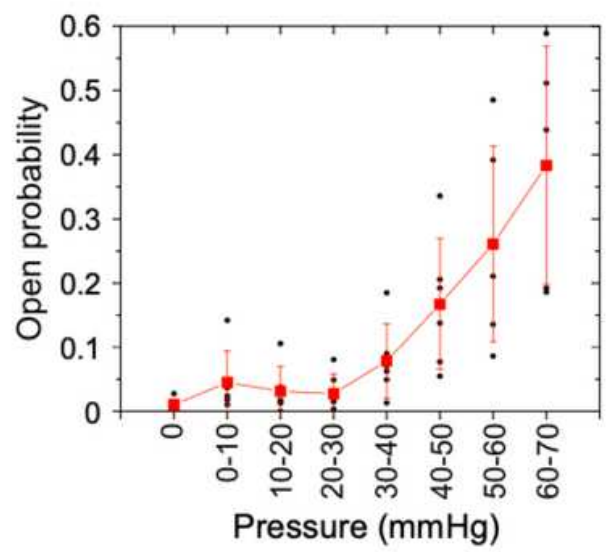

\section{Figure 3}

Channel activities of MCA2(1-173)-6H reconstituted in the lipid bilayer examined by the patch clamp technique. (a) Channel current at a holding potential of 140 to $200 \mathrm{mV}$. (b) Changes in the open probability with the holding potential. (c) Changes in unit current with the holding potential. (d) Channel current (top trace) when a negative pressure (lower trace) was applied. The holding potential was $100 \mathrm{mV}$. (e) Changes in the open probability with the application of negative pressure. Data collected at 140 and $160 \mathrm{mV}$ were combined. In b, c, and e, the mean and standard deviation are shown in red. Small circles indicate individual data. Data from $17(\mathrm{~b}, \mathrm{c})$ and $6(\mathrm{e})$ patches. 
a

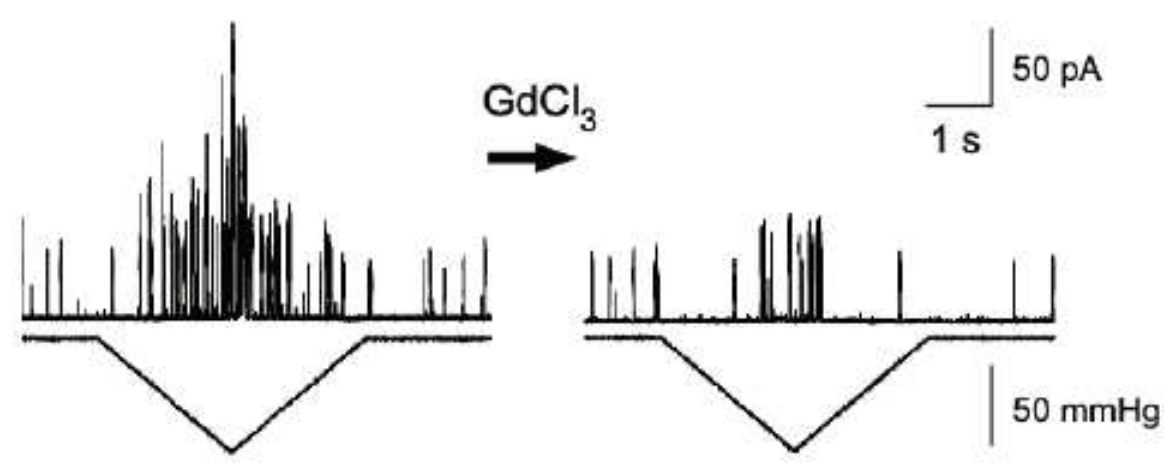

b

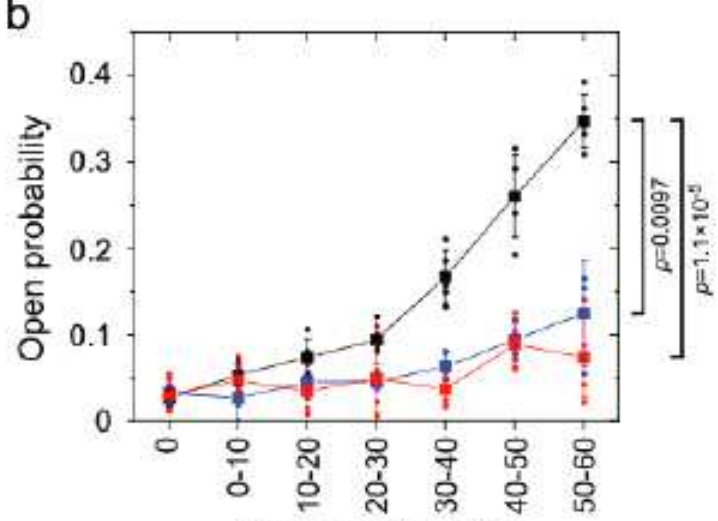

C

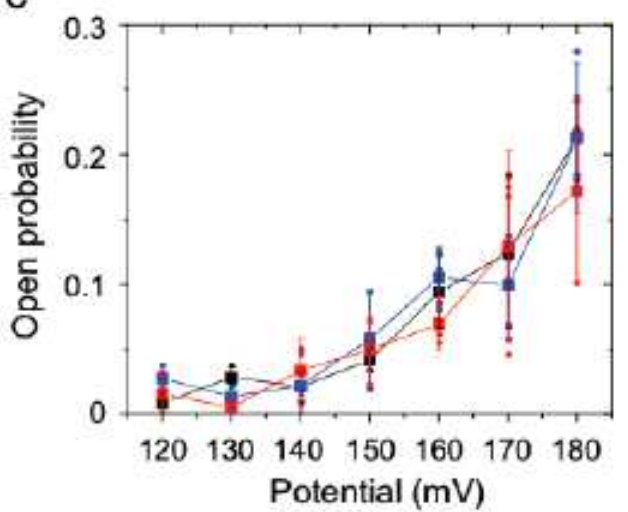

d
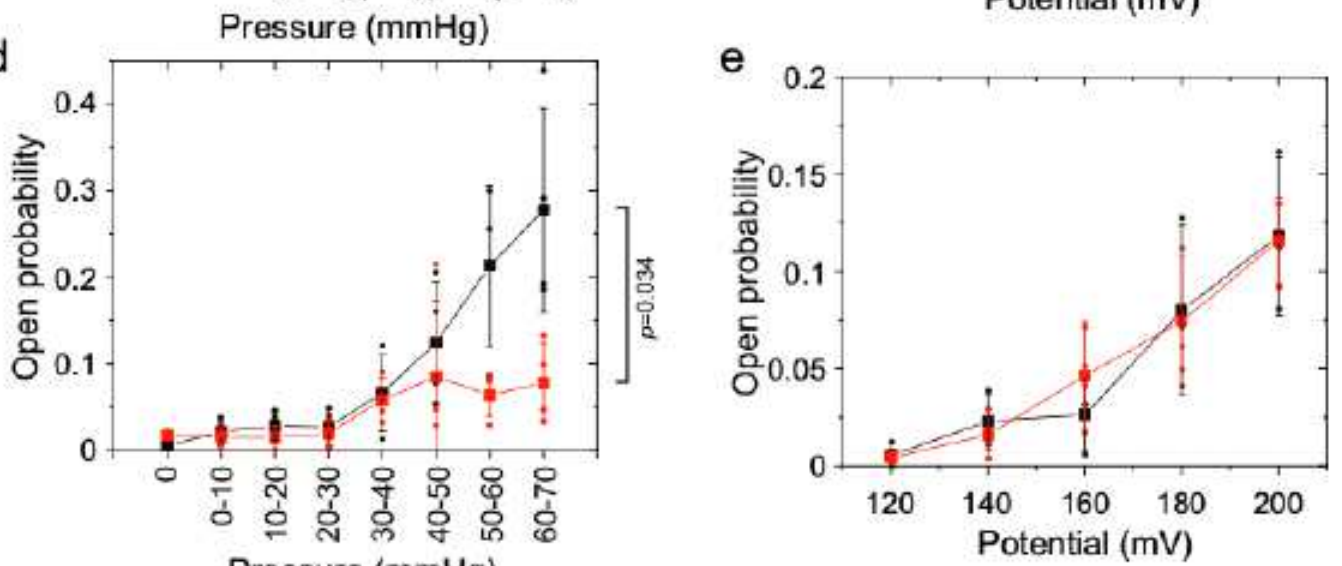

f

Pressure $(\mathrm{mmHg})$

$f$

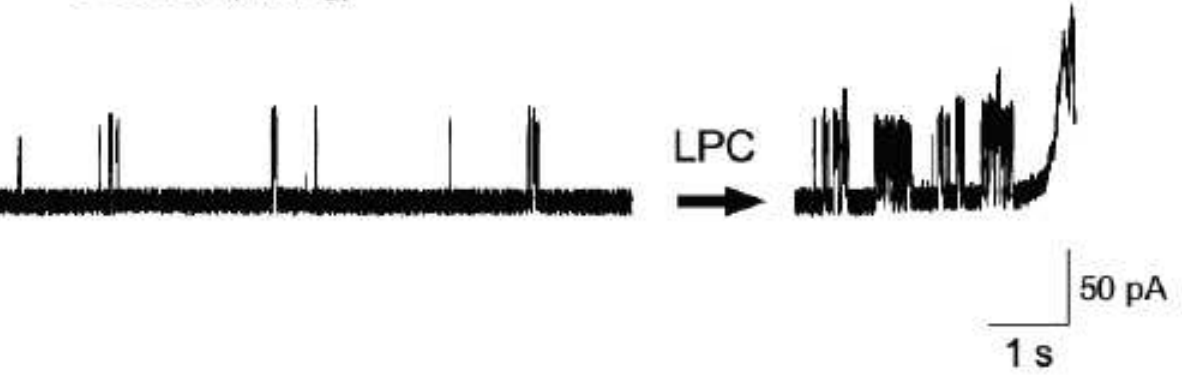

\section{Figure 4}

Effects of mechanosensitive channel blockers on channel activities of MCA2(1- 173)-6H. (a) Negative pressure was applied before (left) and after (right) the application of $30 \mu \mathrm{M} \mathrm{GdCl}$. Current (top trace) and pressure (lower trace) are shown. The holding potential was $140 \mathrm{mV}$. (b, c) Changes in the open probability with pressure (b) and voltage (c) when 0 (black), 10 (blue), or 100 (red) $\mu \mathrm{M} \mathrm{GdCl3} \mathrm{was} \mathrm{applied.}$ $(d, e)$ Changes in the open probability with pressure (d) and voltage (e) when 0 (black) or 5 (red) $\mu \mathrm{M}$ 
GsMT4 was applied. (f) Current traces before (left) and after (right) the application of lysophosphatidylcholine. The membrane was broken at the end of recording. The holding potential was $140 \mathrm{mV}$. In b, c, d, and e, the mean and standard deviation are shown in red. Small circles indicate individual data. Data from 5 (b), 3 (c), 4 (d), and 3 (e) patches. In (b) and (d), p-values of t-test between the data obtained in the presence of blockers and those obtained in the absence are indicated. 